\title{
Eine neue Suchrichtung für die Entwicklungszusammenarbeit
}

\section{Rudolf Högger}

\section{(2) OpenEdition \\ 1 Journals}

Electronic version

URL: http://journals.openedition.org/sjep/1222

DOI: $10.4000 /$ sjep.1222

ISSN: 1663-9677

Publisher

Institut de hautes études internationales et du développement

Printed version

Date of publication: 1 janvier 1991

Number of pages: 255-270

ISSN: 1660-5926

\section{Electronic reference}

Rudolf Högger, «Eine neue Suchrichtung für die Entwicklungszusammenarbeit », Schweizerisches

Jahrbuch für Entwicklungspolitik [Online], 10 | 1991, Online erschienen am: 13 April 2013, abgerufen am 08 September 2020. URL : http://journals.openedition.org/sjep/1222 ; DOI : https://doi.org/10.4000/ sjep. 1222

(c) The Graduate Institute 


\title{
Eine neue Suchrichtung für die Entwicklungszusammenarbeit
}

\author{
Rudolf Högger
}

\section{Einleitung}

"Entwicklung" ist ein normativer, sich verăndernder Begriff, der vieles mitschwingen lässt, was vor allem zeitlich und kulturell bedingt ist. Das Wünschbare prägt inn oft stärker als das empirisch Belegbare. In den fünfziger und sechziger Jahren wurde Entwicklung weitgehend mit wirtschaftlichem Wachstum gleichgesetzt. Spăter kam die Forderung nach sozialem Wandel, d.h. nach Verănderung von Werten, Verhaltensweisen und Machtstrukturen hinzu. Ausgehend von den historischen Erfahrungen Lateinamerikas wurden sodann weltwirtschaftliche Abhängigkeiten als wichtige Ursachen von Unterentwicklung erkannt. Seit den siebziger Jahren werden die kulturelle Eigenstăndigkeit und die Befriedigung der menschlichen Grundbedürfnisse als weitere Schlüsselelemente hervorgehoben. Die jüngsten Postulate richten sich auf Umweltvertrăglichkeit und Nachhaltigkeit (sustainability) der Entwicklung.

Wer sich heute ernsthaft mit Entwicklung und Unterentwicklung beschäftigen will, kommt also nicht darum herum, sich eine nuancierte Vorstellung vom Gegenstand seines Interesses zu machen. Der Zielartikel des schweizerischen Entwicklungshilfe-Gesetzes ${ }^{1}$ deutet dies in wenigen Sätzen an:

1) Die Entwicklungszusammenarbeit unterstützt die Entwicklungsländer im $\mathrm{Be}$ streben, die Lebensbedingungen ihrer Bevölkerung zu verbessern. Sie soll dazu beitragen, dass diese Länder ihre Entwicklung aus eigener Kraft voran-

1) Bundesgesetz über die internationale Entwicklungszusammenarbeit und humanitäre Hilfe vom 19. März 1976, Art. 5. 
treiben. Langfristig erstrebt sie besser ausgewogene Verhältnisse in der Völkergemeinschaft.

2) Sie unterstützt in erster Linie die ärmeren Entwicklungsländer, Regionen und Bevölkerungsgruppen. ...

Die konkreten Lebensbedingungen der Bevőlkerung, die eigene Kraft der Entwicklungslănder und der notwendige Ausgleich in den internationalen Machtund Austauschverhăltnissen werden hier im selben Atemzug angesprochen. Der zweite Absatz misst sodann dem Kampf gegen die Armut ein besonderes Gewicht zu.

Persőnlich halte ich diesen Gesetzestext für eine gute Grundlage staatlicher Entwicklungszusammenarbeit. Sie hat uns bei der $\mathrm{DEH}^{2}$ in den vergangenen Jahren den nötigen Spielraum dafür gegeben, gemeinsam mit Partnerinstitutionen in der Dritten Welt Entwicklungsprojekte zu formulieren, die manchen Aspekten einer komplexen Wirklichkeit Rechnung tragen. Kleinere und grössere Erfolge dieser Bemühungen sind denn auch nicht ausgeblieben.

Trotzdem zweifle ich je lánger desto mehr daran, ob unsere heutige Art, Unterentwicklung und Entwicklung zu verstehen, der lebendigen Wirklichkeit gerecht zu werden vermag. Zu viele unserer gutgemeinten Anstrengungen haben fehlgeschlagen; zuviele Mittel sind wirkungslos versickert, als dass wir die hinter der Entwicklungszusammenarbeit wirkenden Sichtweisen und Planungskonzepte als angemessen betrachten dürften. Insgesamt müssen wir anerkennen, dass Armut, Umweltzerstörung und wirtschaftliche Ungleichgewichte in Entwicklungs- und Industrieländern heute verheerender sind als vor zwei oder drei Jahrzehnten. Ich vermute deshalb, dass das zur Zeit vorherrschende Paradigma von Entwicklung und Unterentwicklung der Realităt nicht in genügend subtiler Weise entspricht und uns somit immer wieder in Sackgassen führt.

Unter einem "Paradigma" verstehe ich jenen Begriff, der durch die Publikation von Thomas S. Kuhn aus dem Jahre 1962 grosse Verbreitung gefunden hat $^{3}$. Verkürzt dargestellt hat Kuhn die These formuliert, dass Wissenschafter und Planer beim Entwurf ihrer Konzepte von ganz bestimmten Annahmen über die Struktur jener Realităt ausgehen, mit der sie sich befassen. Mit dem "Paradigma" sind all die Grundvorstellungen von der Beschaffenheit der Wirklichkeit gemeint, die wir - in unbewusster Voreingenommenheit - in unsere tăgliche Arbeit hineintragen.

Mich beschäftigt also die Frage, ob die Vergeblichkeit so vieler Entwicklungsanstrengungen nicht auch etwas mit dem heute gebräuchlichen Paradigma von Unterentwicklung und Entwicklung zu tun hat. Verfehlen wir die Wirklichkeit vielleicht deshalb so oft, weil wir sie mit einem zu engen, unzeitgemässen Verständnismuster einzufangen versuchen? Natürlich verüge ich über

2) DEH - Direktion für Entwicklungszusammenarbeit und humanitäre Hilfe im Eidgenössischen Departement für auswärtige Angelegenheiten.

3) Thomas S. Kuhn: Die Struktur wissenschaftlicher Revolutionen, Frankfurt a.M. 1973. 
keine gesicherte Antwort auf diese Frage. Meine Zweifel und Vermutungen sind aber stark genug, um wenigstens eine neue Suchrichtung zu umschreiben, die in der Entwicklungszusammenarbeit einzuschlagen wăre.

\section{"Eln multidimensionales Schema von Unterentwicklung" oder: \\ Woran das heutige Paradigma zu erkennen ist}

Um anschaulich zu machen, was nach meinem Verstăndnis die heute vorherrschende Sichtweise von Unterentwicklung und Entwicklung kennzeichnet, greife ich auf ein multidimensionales Schema von Unterentwicklung zurück, dem Handbuch der Dritten Welt von Nohlen und Nuschelert entnommen.

In der Art und Weise, wie dieses Schema einen komplexen Zusammenhang aufzeigt, darf es als Beispiel für die Auffassung vieler heutiger Entwicklungsfachleute gelten. Es dient als Instrument, um sich in einer venwirrlichen Realităt zurechtzufinden. Graphiken wie diese dienen oft als Krücken bei der Planung und bei der Evaluierung von Entwicklungsprojekten.

Welches sind nun die Grundvorstellungen von Entwicklung und Unterentwicklung, die sich mit einer solchen Darstellungsweise verbinden? Ich will bei der Beantwortung dieser Frage nicht auf einzelne Kästen, Begriffe oder Wirkungspfeile in der Graphik eingehen (über welche die Fachleute im Einzelnen ohnehin lange zu streiten vermöchten). Es geht mir vielmehr darum, einige grundsătzliche, für viele ăhnliche Schemata gültige Gesichtspunkte herauszuarbeiten.

Das vorliegende Schema von Unterentwicklung ist zunächst nichts anderes als eine Auslegeordnung jener Entwicklungsfaktoren, mit welchen sich die heutigen Fachleute auseinandersetzen. Neben internen (volkswirtschaftlichen und sozialen) Faktoren werden externe (international wirkende) Krăfte ausgemacht; zu okonomischen Gesichtspunkten treten gesellschaftliche und individuelle Werte; Begriffe wie "Arbeitsproduktivităt" oder "Importtechnologie" werden ergănzt durch Ausdrücke wie "Kontemplation" oder gar "Schönheit". Dass das hier aufgereihte Gedankengut "multidimensional" ist, lässt sich in der Tat kaum bestreiten.

Die "Mehrdimensionalităt" scheint mir ein erstes wichtiges Merkmal des heutigen Paradigmas zu sein. Es gibt in der Entwicklungszusammenarbeit nichts, was für unsere Ziele nicht potentiell wichtig sein könnte und womit wir uns folglich nicht beschăftigen müssten: vom Brotkorb der Armen bis zum Verwaltungsreglement, vom Basisgesundheitsdienst bis zu Wirtschaftskonzepten mit wohlangepassten Stützprogrammen. Und es gehört zu unserem Selbstverstăndnis,

4) Dieter Nohlen/Franz Nuscheler: Handbuch der Dritten Welt, Band 1: Unterentwicklung und Entwicklung; Theorien - Strategien - Indikatoren; Hamburg 1982, S. 46. 

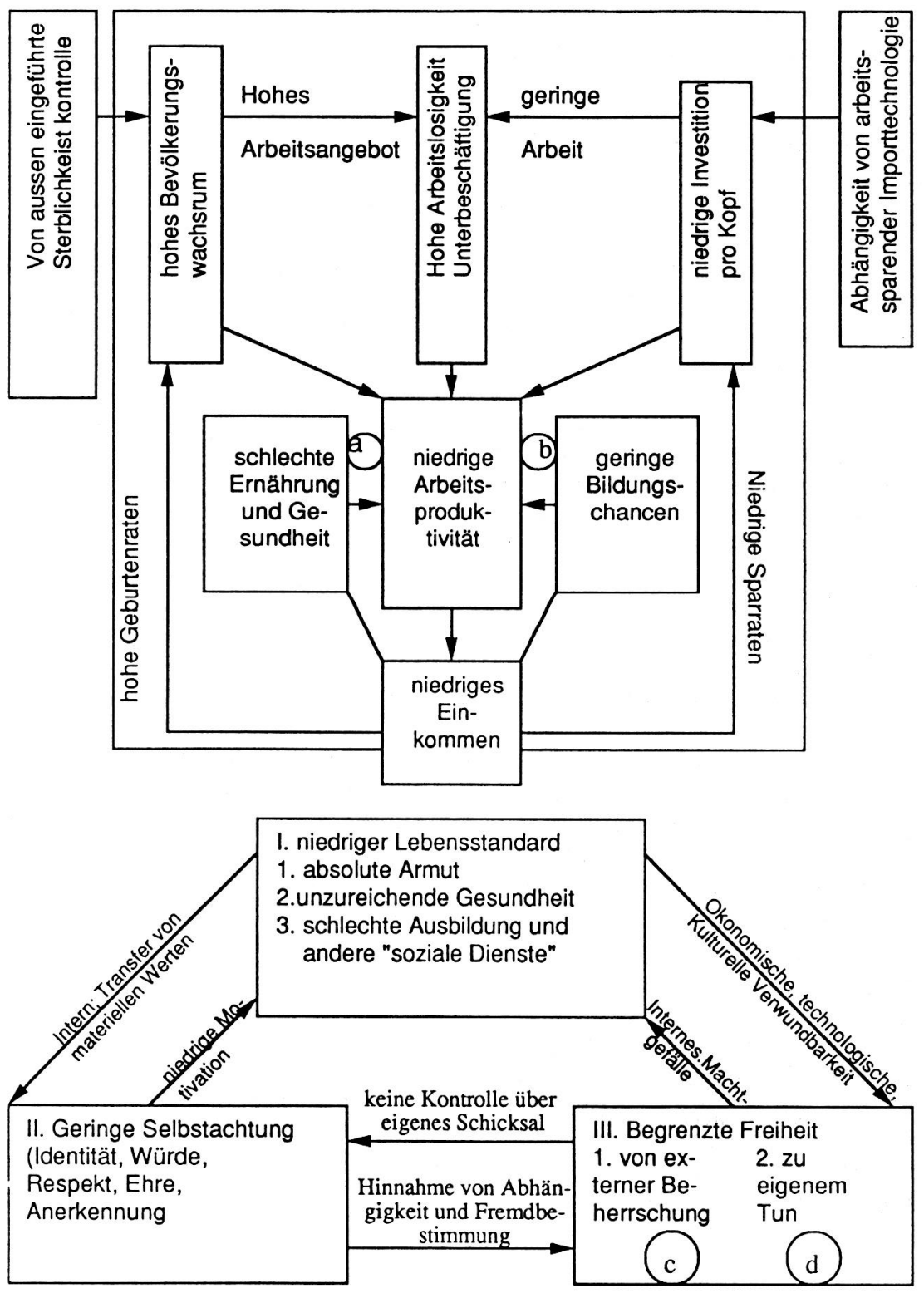

$\mathrm{a}=$ niedrige Arbeitsmotivation $\mathrm{b}=$ unzulängliche Managemenfähigkeiten

$c=$ Handẹl, private und öffentliche Hilfe, Technologie Bildung, Wertte und Lebensstil

$\mathrm{d}=$ materieller Gewinn, Musse, Kontemplation, Schönheit, Lebensstil 
mit all diesen Dimensionen vertraut zu sein, sie auf dem Diskussionstisch auszubreiten und zu versuchen, ihnen in der tăglichen Arbeit Rechnung zu tragen.

Ein zweites Merkmal der heutigen Verstăndnisweise von Entwicklungsprozessen wird an unseren Bemühungen deutlich, die Dinge auseinanderzuhalten. Das kommt in den Klarheit schaffenden Kăsten und Rahmen des vorliegenden Schaubildes zum Ausdruck. Die gleiche Sorge um saubere Gliederung spiegelt sich aber auch in zahllosen Inhaltsverzeichnissen von Planungs- oder Evaluierungsberichten. Naturrăumliche Grunddaten werden von den Eckwerten der Volkswirtschaft oder des politischen Systems abgesondert. Kulturelle Anliegen dürfen den Blick für aussenwirtschaftliche Realităten nicht trüben; und Begriffe wie "Sterblichkeitskontrolle", "Investitionen", "Selbstachtung" oder "Freiheit" gehören in je separate Gedankenkăsten, damit aber auch in unterschiedliche Ecken der Gesamtvorstellung (siehe dazu Schaubild 1).

Selbstverstăndlich bleiben wir uns bewusst, dass die einzelnen Kräfte, Faktoren oder Elemente der Entwicklung in Wirklichkeit miteinander verbunden sind und einander gegenseitig beeinflussen. Der Wille, vernetzt zu denken, wird sehr betont, und dies scheint mir ein drittes entscheidendes Merkmal des gültigen Paradigmas zu sein. Wir verstehen die Vorgänge in der Dritten Welt längst nicht mehr als statische Bedingtheiten von einigen Ursachen und Wirkungen, sondern als dynamischen Zusammenhang sehr unterschiedlicher, ja gegenläufiger Krăfte. Sie zu meistern ist lăngst keine handwerkliche Aufgabe mehr, sondern ein Thema anspruchsvollster "High-Tech".

Das lässt sich in unserem Schema an den vielen Wirkungspfeilen und ihren verschiedenen Richtungen ablesen. Dabei sind die so angedeuteten Bewegungen innerhalb des Systems nicht nur aus linearer Kausalität, sondern oft auch aus gegenläufigen Rückkoppelungen oder zirkulären Verstärkungen zu verstehen. Dahinter steht ein dynamisches und vor allem systemisches Verständnis von Wirklichkeit. Im Alltag der Entwicklungsfachleute spiegelt es sich in dem immer neu formulierten Anspruch, interdisziplinär arbeiten zu wollen. Oft führt es allerdings zu wenig schlüssigen, hitzigen Debatten über die Funktionsweise des Ganzen, die von niemandem endgültig durchschaut wird.

Zusammenfassend und verkürzt möchte ich das Paradigma, das zur Zeit in der Entwicklungszusammenarbeit vorherrscht, etwa folgendermassen umreissen: Wir betrachten Entwicklung und Unterentwicklung als komplexes Zusammenspiel von sehr unterschiedlichen, oft auch gegensätzlichen Kräften, die letzten Endes wie die Rädchen, Wellen und Hebel einer Maschine, vielleicht noch eher wie die Mikro-Chips eines Computers ineinander wirken. Es wäre zu einfach, dieses Paradigma als mechanistisch zu bezeichnen, aber es bleibt ohne Zweifel von technischen - von modernsten technologischen - Vorstellungen bestimmt. Täglich erfahren wir, wie anspruchsvoll es ist, die Funktionsweise des Ganzen zu erfassen, aber im Grunde genommen zweifeln wir nicht daran, dass es grundsätzlich möglich sein muss, das Gesamtsystem nicht nur gedanklich abzubilden, sondern auch in die von uns gewünschte Richtung zu steuern.

Natürlich sind wir uns selber gegenüber auch kritisch, aber diese Kritik betrifft in der Regel unsere gelegentlich mangelnde Sorgfalt, unsere oft ungenü- 
genden Fachkenntnisse in Einzelfragen oder unsere Unfăhigkeit, wirklich interdisziplinăr zu arbeiten. Die allgemein vorherrschende grundsătzliche Sichtweise selber steht kaum je zur Debatte. Gerade dadurch enweist sie sich als jenes Paradigma, das unsere Überlegungen und Tătigkeiten am stărksten - weil am wenigsten bewusst - prăgt.

\section{Ein Blick in den Alltag der Entwicklungspolitik oder: Dle Vorzáge und die Schwáchen des heutigen Paradigmas}

Bevor ich mich kritisch mit unserem heutigen Entwicklungsverstăndnis auseinandersetze, müssen - trotz allem - dessen Vorzüge hervorgehoben werden. Das ist deshalb wichtig, weil es hier nicht darum geht, "das richtige" Paradigma oder neue Patentlösungen für die Probleme der Unterentwicklung anzubieten. Ich bin dazu nicht in der Lage, und ich nehme an, dass solche Rezepte auch sonst nirgends wohlfeil zu haben sind. Wir bleiben auf vieles angewiesen, was in den vergangenen Jahrzehnten an Konzepten und Methoden erarbeitet worden ist. Auch wenn es uns gelingt, allmăhlich in ein enweitertes Paradigma hineinzuwachsen, wird manches Element der heutigen Verstăndnisweise weiterhin hilfreich und notwendig bleiben.

Als wichtige Merkmale des derzeitigen Paradigmas habe ich die "Mehrdimensionalităt", die "Differenziertheit" und die "Vernetztheit" der Entwicklungskrăfte hervorgehoben. Alle drei sind für die Bewăltigung heutiger Probleme unerlässlich und müssen weiterhin Teil des Instrumentariums in der EZ bleiben. So wird die Bereitschaft zu multidimensionalem Denken helfen, in der politischen Diskussion etwa um die Verschuldungskrise, um Entwicklungshilfe-Kredite oder um Asylfragen den Verlockungen bequemer Vereinfachung zu widerstehen. In der Verschuldungskrise kreuzen sich ja tatsăchlich nationalstaatliche und internationale, soziale und wirtschaftliche Problemstrănge; die Entwicklungszusammenarbeit muss unter innen- wie aussenpolitischen, technischen und kulturellen Gesichtspunkten angegangen werden; und im Streit um die Ausgestaltung oder Einschränkung des Asylrechts gilt es, konservative Zurückhaltung, irrationale Ängste und stürmisch humanitäre Anliegen zu versőhnen. Mehrdimensionalităt ist unerlässlich, auch wenn sie uns oft sehr belastet.

Umgekehrt müssen wir uns immer auch dagegen wehren, im Wust der sich widersprechenden Gesichtspunkte die Orientierung zu verlieren und uns von ungeklärten Gefühls- oder Argumenten-Strömen fortschwemmen zu lassen. Kritische Differenzierung ist die Voraussetzung für nüchternes Abwăgen: Interne Mechanismen der Entwicklungslănder müssen von externen Krăften abgehoben werden; soziale Gesichtspunkte lassen sich nicht einfach ökonomischen Sachzwängen unterordnen; materieller Wohlstand kann auch nicht notwendigerweise auf Fleiss oder Können zurückgeführt werden. Manche Entwicklungsplanung wăre realistischer ausgefallen, manches Vorurteil nicht weitergegeben worden, wenn wir die verschiedenen Wirkkrăfte klarer von einander getrennt und auf ihre Eigengesetzlichkeit hin geprüft hätten. 
Manche Planung wăre andererseits erfolgreicher verlaufen, wenn es gelungen wăre, die Zusammenhănge deutlicher zu sehen und das Netz der Bezüge zwischen allen Einzelfaktoren in Betracht zu ziehen. Wie oft stellen wir im nachhinein fest, dass ein Entwicklungsprojekt vor allem deshalb sein Ziel verfehit hat, weil die Bedeutung irgend eines einzelnen Elements - Betriebswirtschaftlichkeit, Anfälligkeit aư Krankheit oder soziale Rangordnung - vernachlăssigt wurde. Unser technizistisches Paradigma ist zu eng, als dass es uns einen ganzheitlichen Zugang zur Lebenswirklichkeit von Unterentwicklung und Entwicklung vermitteln könnte.

Diese Kritik werde ich in den năchsten Kapiteln anhand konkreter Beispiele zu vertiefen suchen. Die ersten Zweifel am heutigen Paradigma ergeben sich im Grunde aber schon aus dem "multidimensionalen Schema" von Nohlen und Nuscheler. Hier fălt nămlich die merkwürdige Künstlichkeit auf, mit welcher beispielsweise ein "hohes Bevölkenungswachstum" mit "Identităt" und mit "Würde" des Menschen vernetzt bzw. nicht innerlich verbunden ist - so als ob die Zeugung von Nachkommenschaft nichts oder wenig mit der persönlichen oder familiären Identität zu tun hätte, nicht mit der Würde von Mutter- und Vaterschaft in eins fiele. In diesem Punkt wirkt das von "High Technology-Denken" geprăgte Paradigma plötzlich merkwürdig hilflos.

Gewiss ist das statistische Phänomen des Bevölkerungswachstums ein anderer Aspekt der Unterentwicklung als Identităt und Würde es sind. Gewiss ist die erstgenannte Erscheinung quantifizierbar und von hoher volkswirtschaftlicher und ökologischer Relevanz. "Identität" und "Würde" dagegen fallen nicht in den Bereich dieser Wissenschaften, sondem müssten allenfalls der Philosophie, Psychologie, Ethik oder Religionswissenschaft zugeordnet werden. Differenzierung im Sinne gedanklicher Unterscheidung ist also angezeigt. Ist es aber zulăssig, die so unterschiedenen verschiedenen Aspekte eines unteilbaren Ereignisses wie der Geburt eines Kindes schematisch von einander zu trennen, wie das mit Bestandteilen einer Maschine oder eines Computers, ja mit den Elementen einer chemischen Verbindung gemacht werden kann? Lassen sich die Einzelaspekte eines ganzheitlichen Vorgangs nach verschiedenen wissenschaftlichen Disziplinen gesondert behandeln und spăter in ein auf Kausalităt beruhendes Bezugsnetz wiedereingliedern? Gibt es eine wissenschaftliche Logik, nach welcher das quantitative Bevölkerungswachstum mit nicht quantifizierbaren menschlichen Haltungen oder Werten "vernetzt" werden kann?

Ich vermute stark, dass dies nicht der Fall ist. Viel eher geraten wir hier in den Bereich hilfloser Willkür, die anzeigen könnte, dass unser Paradigma dem Leben in entscheidenden Punkten nicht gerecht zu werden vermag.

Dle "Bedürfnispyramide von Maslow" oder:

Das herkơmmliche Paradigma führt in die Sackgasse

Was sich anhand des bisher betrachteten Schemas vermuten liess, wird deutlicher, wenn wir eine in der Entwicklungszusammenarbeit verbreitete und be- 
liebte Theorie der Handlungsmotivation als neues Beispiel heranziehen. Es handelt sich um das Modell der Bedürnnishierarchie des amerikanischen Psychologen Maslow ${ }^{5}$. Wie sehr sie Ausdruck des dominierenden Paradigmas ist, lăsst sich daran ablesen, dass ihre gute Verstăndlichkeit, ihre Plausibilităt und praktische Verwendbarkeit in Arbeitshilfen für Entwicklungsfachleute immer wieder hervorgehoben wirdt.

Maslow glaubt, in der Art, wie Menschen ihre verschiedenen Bedürfnisse zu befriedigen suchen, drei Gesetzmăssigkeiten unterscheiden zu kőnnen:

1) Jeder Mensch trachtet danach, seine Bedürfnisse systematisch zu befriedigen, angefangen mit den elementarsten (physiologischen) bis zu den hierarchisch höchsten Bedürfnissen (Selbstverwirklichung);

2) Das Verhalten des Menschen wird durch das jeweils elementarste nicht befriedigte Bedürfnis bestimmt;

3) Hierarchisch tiefere Bedürfnisse drängen, wenn sie unbefriedigt sind, stärker nach Befriedigung als höhere Bedürfnisse.

In dieser Theorie begegnen wir allen früher beschriebenen Hauptmerkmalen unseres Entwicklungs-Paradigmas ( $v g l$. dazu die graphische Darstellung auf der folgenden Seite): Die komplexe Ganzheit des Menschen und seiner Bedürttigkeit wird nach unterschiedlichen Aspekten gegliedert. Es entsteht eine auf den ersten Blick plausibel anmutende Auslegeordnung, welche differenzierte Untersuchungen nach den Kriterien verschiedener wissenschaftlicher Disziplinen möglich macht. Das ist nützlich. Abklärungen über den tăglichen Năhrstoff- und Kalorienbedarf eines Menschen, einer Familie, eines Bevölkerungsteils etc. liefern unentbehrliche Unterlagen für die landwirtschaftliche Planung oder die Vorbereitung von Nothilfeaktionen.

Gleichzeitig wird deutlich gemacht, dass auch mit der besten Grundversorgung, mit der vollständigsten Deckung physiologischer Grundbedürfnisse noch längst nicht alle Probleme von Armut und Unterentwicklung gelöst sind. Soziale Bedürfnisse oder die individuelle Suche nach Wertschătzung wollen zusătzlich und in ganz anderer Weise respektiert werden als der physikalische oder chemische Bedarf. Entwicklungszusammenarbeit muss sich - wie wir schon betonten - differenzierter orientieren als an Mengenproblemen.

Maslow versucht überdies, die vielfältig aufgefăcherten Einzelbedürnnisse des Menschen in einer hierarchischen Ordnung zu vernetzen, aus welcher sich kausale Erklärungen für bestimmte Verhaltensweisen ableiten lassen. Der systemische Charakter des Modells wird dadurch besonders deutlich. Mit dieser

5) Die Theorie von Maslow wird hier zusammengefasst nach: Vreni Baggenstoss und Rolf Gubser: Betroffene zu Beteiligten machen; Studienarbeit IAP, 1981.

6) Vgl. z.B. Harald Lang: Management der Projektdurchführung im Partnerland; ein Leitfaden; hrsg. Deutsche Gesellschaft für Technische Zusammenarbeit (GTZ), Rossdorf 1987. 


\section{Bedürfnis nach Selbstverwirklichung}

Entwicklung der eigenen Persön-

lichkeit, Kreativität, Selbstentfaltung

\section{Bedürfnis nach Wertschătzung}

Selbstvertrauen, Selbstachtung, Wunsch nach Erfolg,

Stärke, Macht und Anerkennung. Prestige, Status,

Unterscheidung von anderen

\section{Soziale Bedürnisse}

Bedürnis nach Zugehörigkeit zu einer sozialen Gruppe, danach, von anderen akzeptiert zu werden, Freundschaft, Liebe. Vermeiden von Ablehnung und Einsamkeit

\section{Bedürfnis nach Sicherheit}

Beseitigung von Gefahren für die materielle Existenz wie Schutz vor Krankheit, Alter, Arbeitslosigkeit, Bedürnnis nach Ordnung, Stabilität, Zuverlässigkeit

\section{Physiologische Bedürnisse}

Essen, Trinken, Schlafen, Sexualität, Schutz vor der Witterung, Bedürfnis nach Atemluft und Wahrnehmungsreizen

Darstellungs- und Verständnisweise scheinen sich auch die gesuchten Ansatzpunkte für steuernde menschliche Eingriffe ins Ganze der menschlichen Bedürftigkeit zu ergeben. Maslow bestătigt mit seiner Pyramide den gesunden Menschenverstand, dem es rasch einleuchtet, dass ein Projektmitarbeiter langfristig nicht durch Erfolg und Anerkennung (Bedürnnis nach Selbstbestätigung) motiviert werden kann, wenn dessen Hauptsorge darin besteht, seine Familie knapp zu ernähren (physiologische Bedürfnisse) oder wenn er um die Erhaltung seines Arbeitsplatzes bei Projekt-Ende bangen muss (Bedürnis nach Sicherheit) ${ }^{7}$. Eine derartige Analyse muss den Projektmanager zu klaren Massnahmen veranlassen.

An diesem Punkt wird aber auch die Gefahr einer unglaublich verkürzten Sichtweise spürbar. Das bereits früher angesprochene Beispiel der menschlichen Fortpflanzung (die hinter dem Problem der "Bevölkerungs-Explosion" steht) bringt dies besonders krass zum Ausdruck: Maslow trennt den physiologi-

7) a.a.O. S. 147 . 
schen Trieb der Sexualităt beispielsweise vom menschlichen Bedürfnis nach Zuverlăssigkeit (zweite Ebene), von der Sehnsucht nach Liebe (dritte Ebene), der Furcht vor Einsamkeit (dritte Ebene), dem Verlangen nach Selbstachtung (vierte Ebene) und Selbstentfaltung (fünfte Eben). Alle diese Aspekte des Eros werden verschiedenen hierarchischen Stufen zugeordnet. Da nun das Verhalten des Menschen - nach Maslow - durch das jeweils elementarste nicht befriedigte Bedürfnis bestimmt wird, muss wohl angenommen werden, dass sich die Slum-Bewohner von Bombay, Abidjan oder Bogotà in ihrem tăglichen Kampf um das Überleben kaum von "höheren" Bedürfnissen leiten lassen. Partnerschaftliche Zuverlăssigkeit, Liebe, Freundschaft oder Wărme sind innen wohl unbekannt oder unwichtig - von Selbstvertrauen oder Selbstachtung und einer eigenstăndigen Persónlichkeit gar nicht zu reden.

Indem wir "Liebe", "Selbstachtung" und "Persönlichkeit" von "rein physiologischer Sexualität" abspalten, verstümmeln wir jene Menschen, die um ihre physiologische Existenz kămpfen müssen. Wir trennen sie von ihrer eigenen seelischen Wirklichkeit; schlimmer: eine solche Wirklichkeit wird den Ärmsten der Welt abgesprochen.

Dass eine solche Sichtweise der Realität in der Dritten Welt in keiner Weise gerecht zu werden vermag, spürt jede(r), der (die) schon mit materiell sehr armen Menschen in Entwicklungslăndern in direktem Kontakt gestanden hat.

Mit Theorien wie der Maslow'schen Bedürfnispyramide liefert unser sich wissenschaftlich gebärdendes Paradigma fatale Rechtfertigungen dafür, die Benachteiligten in allen Teilen der Welt weiter zu erniedrigen. Die Prostituierte in Bangkok, von deren Erwerb mehrere Familienangehörige leben, "hat ja wohl keine anderen Bedürnnisse". Bei einer solchen Perspektive wurzelt auch die Apartheidspolitik "in natürlichen Gegebenheiten"; und die Tatsache, dass wir von Billiglohnländern profitieren, hängt dementsprechend mit dem Umstand zusammen, "dass für die dortigen Frauen und Mănner eine Săttigung auf physiologischer Ebene ausreicht", wăhrend sich der schweizerische Arbeiter "offenbar den Luxus von Persönlichkeitsbildung und Selbstentfaltung leistet".

All dies mag übertrieben und polemisch klingen. Möglicherweise wird es auch den bewussten Auffassungen von Maslow nicht gerecht. Die Überspitzung macht aber den kritischen Punkt sichtbar, an welchem die Stärken unseres $\mathrm{Pa}$ radigmas - Mehrdimensionalität, Differenzierung und Vernetzung - in unannehmbare Plumpheit umkippen. Es ist der Punkt, an welchem wir spüren, dass wir der menschlichen Wirklichkeit weder durch weiteres Differenzieren noch durch immer raffinierteres Vernetzen năher kommen, sondern auf einen neuen Ansatz, ein frisches Gespür für eine ganzheitliche, nicht zerlegbare Wirklichkeit angewiesen sind.

Offenbar ist es so, dass die Methode des Trennens und Vernetzens weniger weit reicht, als in den ersten drei Entwicklungsjahrzehnten angenommen. Sie ist überall da hilfreich, wo es um die Erfassung vordergründiger Zusammenhänge, um chemische, physikalische oder physiologische Prozesse, um Güterströme und Geldflüsse, nicht zuletzt auch um naturräumliche Veränderungen geht. Sie wird aber zunehmend fragwürdig, je komplexer die zu erfassende Wirklichkeit 
ist, wobei komplex nicht einfach ein besserer Ausdruck für "sehr kompliziert" ist. Komplexităt verstehe ich als die Ganzheit der genannten ausseren Wirklichkeiten und der inneren Wirklichkeiten der menschlichen Psyche. Mit anderen Worten: Unser gegenwärtiges Paradigma kann dort nicht wirklich weiter helfen, wo es danum geht, den Menschen oder die Gesellschaft nicht als superkomplizierte Maschine, sondern als körpenlich-seelische Ganzheit zu verstehen.

\section{Strukturanpassungsprogramme in Afrika oder: Die Notwendigkelt elner neuen Orlentlerung}

Als Beispiel für unsere Überlegungen soll Madagaskar dienen. Das Land und seine Bevölkenungsmehrheit befinden sich in einer verzweifelten Situation (siehe Kasten). Die wachsende Zahl von Bettlem ist in diesem Inselstaat als Folge und Indiz der gravierenden Schuldenkrise zu betrachten. Über drei einhalb Milliarden Dollar betrăgt die gesamte Aussenschuld Madagaskars, und selbst nach fünf aufeinanderfolgenden international vereinbarten Umschuldungen muss das Land noch immer mehr als die Hälfte seiner Exporterlöse für den Schuldendienst aufwenden. Was Madagaskar auf dem Weltmarkt anzubieten hat, ist immer weniger gefragt und erzielt nur niedrige Preise.

Zum Beispiel Madagaskar: Nach Angaben der Weltbank haben die Gesundheitsausgaben des Staates von Fr. 10.70 pro Kopf der Bevölkerung im Jahre 1980 auf Fr. 2.701987 abgenommen. Weil bisher kaum Personal entlassen wurde, sparte man vor allem bei den Medikamenten. Nur während 2-3 von 12 Monaten im Jahr verfügen die Gesundheitsstationen über einen Vorrat an Basismedikamenten. Während 9-10 Monaten werden die Patienten in die private Apotheke geschickt, deren Gestelle zwar voll, deren Preise für die Durchschnittsmadegassen aber unerschwinglich sind. Um die Mangellage an Medikamenten etwas zu lindern, hat die Schweiz einen Beitrag von 10 Millionen Franken geleistet, mit dem unter anderem Chloroquin zur Bekämpfung der Malaria finanziert worden ist.

An sich hatte Madagaskar während Jahren eine sorgsame Finanzpolitik betrieben. Das änderte sich jedoch 1978 schlagartig, als der Traum vom Industriestaat in die Wirklichkeit umgesetzt werden sollte. Über Nacht lancierte die Regierung Dutzende von ehrgeizigen Projekten und nahm dafür Auslandskredite auf. Der Traum vom Sprung nach vorne, Korruption, fallende Rohstoffpreise und ausländische, auf rasches Geld erpichte Geschäftsleute trieben Madagaskar in die Schuldenfalle.

Heute besinnt man sich in Madagaskar wieder auf den Agrarstaat. Seit 1980 arbeitet die Regierung eng mit dem Internationalen Währungsfonds und der Weltbank zusammen und lässt sich von ihnen beraten. Weil Madagaskar den Rezepten aus Washington seit Jahren folgt, hat sich die Insel sogar den Ruf eines Musterschülers eingehandelt. Doch noch immer ist kein Ende des Tunnels in Sicht. Trotz allen Sanierungsbemühungen muss jeder zweite im Export verdiente Franken für den Schuldendienst aufgewendet werden. Und das bei einem Volkseinkommen, das sich pro Kopf der Bevölkerung von 350 Dollar im Jahr 1980 auf 210 
Dollar (1987) verringert hat. Zum Vergleich: In der Schweiz ist das durchschnittliche Volkseinkommen in derselben Zeitperiode von 16'440 auf 21'250 Dollar gestiegen.

Quelle: Arbeitsgemeinschaft Swissaid, Fastenopfer, Brot für Brüder und Helvetas.

Beraten und finanziell unterstützt von internationalen Institutionen und bilateralen Hilfsgebern (auch der Schweiz) unterzieht sich das verarmte Land seit Jahren einer langen Reihe von sogenannten Strukturanpassungsprogrammen. Das Ziel dieser Sanierungsmassnahmen ist es, die internationale Kreditwürdigkeit des Schuldners wiederherzustellen, indem ein neues Gleichgewicht zwischen den finanziellen Verpflichtungen nach aussen und den inneren wirtschaftlichen Möglichkeiten hergestellt wird. Das effordert unter anderem Massnahmen auf dem Gebiet der Wăhrung und jenem der Ordnungspolitik (mehr Spielraum für privatwirtschaftliche Initiative), insbesondere aber einschneidende Sparmassnahmen, unter denen vor allem die ohnehin benachteiligten Schichten zu leiden haben, sowie einen Abbau staatlicher Strukturen. Die Reallöhne sind in den vergangenen sieben Jahren um rund $60 \%$ gesunken. In ăhnlichem Ausmass haben sich der Bestand und die Gestaltungsmöglichkeiten der Verwaltung reduziert.

Strukturanpassungsprogramme sind besonders deutlich von jenem entwicklungspolitischen Paradigma geprägt, dessen Hauptmerkmale wir als "Mehrdimensionalität", "Differenziertheit" und "Vernetztheit" kennen gelernt haben. Massnahmen zur strukturellen Anpassung einer Volkswirtschaft fussen auf der Annahme, dass es möglich und sinnvoll ist, steuernd in das "unterentwickelte" politisch-ökonomische Gefüge einzugreifen und damit das weitere Geschehen unter Kontrolle zu bringen. Ich betone noch einmal, dass eine solche Sichtweise in mancherlei Beziehung gerechtfertigt ist und durch zahlreiche Erfahrungen empirisch belegt werden kann. Nicht zufällig folgt Madagaskar und folgen viele afrikanische Staaten den so begründeten Ratschlägen des Internationalen Wăhrungsfonds und der Weltbank. Es ist in der Tat schwierig, grundsätzlich andere wirtschaftlich-politische Konzepte vorzulegen, die einer ökonomischen Gesundung der hochverschuldeten Entwicklungsländer unter den heutigen weltweiten Rahmenbedingungen dienlich wären.

Trotzdem befürchte ich, dass die vordergründig enfolgreichen systembeherrschenden Techniken, die der Weltwirtschaft heute ihr Geprăge geben, dem Hauptanliegen einer längerfristigen und umfassenden Entwicklung nicht genügend gerecht werden. Vieles spricht dafür, dass die nach dem heutigen Paradigma forcierte "Sanierung" oder "Entwicklung" der Dritten Welt nicht nachhaltig sein wird. Unter Nachhaltigkeit (sustainability) verstehe ich ein sehr delikates Gewebe von ganz unterschiedlichen Voraussetzungen, die erfüllt sein müssen, damit sich Entwicklung dauerhaft und von innen heraus, ohne stetige patronisierende und subventionierende Aussenbeiträge vollziehen kann. "Von innen heraus", das heisst: auf Grund eigener Wirtschaftskräfte, eigenen Wissens, eigener Geschichte und eigenen Bewusstseins - vor allem aber eigener innerer Be- 
ziehung zum ăusseren materiellen Fortschritt. Die stetige Herausforderung durch Äusseres und Fremdes wird dadurch nicht aufgehoben, wohl aber ins Gleichgewicht mit dem Eigenen und Inneren gebracht.

Diesem notwendigen, lebens- und entwicklungsfördernden Gleichgewicht ist mit den heutigen Strukturanpassungsprogrammen schlecht gedient. Der Koordinator der schweizerischen Entwicklungshilfe in Madagaskar wird sich dieser Tatsache immer von neuem bewusst, wenn er mit Vertretern der Verwaltung oder mit Ministern Gesprăche führt. Das Schuldenproblem drăngt wăhrend vieler solcher Kontakte in den Vordergrund. Die Madagassen empfinden es wie eine würgende Hand an der eigenen Kehle. Das Erstickende dabei ist aber nicht nur die massive materielle Einschrănkung, die mit den Strukturanpassungen verbunden ist, sondern ebenso sehr der Verlust an nationaler und persőnlicher Souverănităt. "Wir stehen mit dem Rücken zur Wand, sind nicht mehr Meister im eigenen Haus," geben sie dem hörbereiten Partner zu verstehen. Und dieser spürt: "Gerade weil die Sachlogik der Strukturanpassungen so zwingend erscheint, ist die Bitterkeit über die damit verbundene Entwürdigung so tief."

Rwandas Präsident Juvenal Habyarimana hat dieses Gefühl der Würdelosigkeit aufgenommen und inm vor einer Versammlung afrikanischer Staatsund Regierungschefs im Herbst 1987 öffentlich Ausdruck verliehen ${ }^{8}$. Für ihn gibt es keinen Zweifel, dass es ohne menschliche und nationale Würde keine nachhaltige, von innen mitbestimmte Entwicklung geben kann; oder umgekehrt: dass solche Würde nur die Frucht einer inneren Beziehung und Mitverantwortung gegenüber dem politischen und ökonomischen Geschehen sein kann. Die Strukturanpassungsprogramme und das dahinter stehende technizistische $\mathrm{Pa}$ radigma (worin materieller Fortschritt und seelische Entwicklung getrennt betrachtet und "behandelt" werden) sind offenbar nicht in der Lage, Wirtschaftsreform und Würde als zwei Aspekte einer einzigen, ganzheitlichen Wirklichkeit zu verstehen.

Ich möchte diesen Punkt mit einem Bild verdeutlichen: Wirtschaftsreform und Würde sind für den afrikanischen Staatsmann wie die Vorder- und die Rückseite einer Hand. Betrachten Sie Ihre Hand, wenden Sie sie, nehmen Sie die beiden Seiten getrennt wahr. Sie haben es mit zwei Aspekten einer einzigen Realităt zu tun. Es ist nicht möglich, Vorder- und Rückseite mit dem Chirurgenmesser von einander zu trennen. Wenn Sie es trotzdem versuchen sollten, hätten Sie es mit zwei halben Händen, nicht mit einer Vorder- und einer Rückseite zu tun. Genau so verhält es sich nach Habyarimana mit der Ganzheit von Wirtschaftsreform und Würde.

Was hiesse es denn aber, wenigstens einen ersten Schritt auf diese Ganzheit hin zu unternehmen? Habyarimana fasst zwei unterschiedliche, sich aber notwendig ergänzende Bewegungen ins Auge. Die erste muss von den Afrikanern selber eingeleitet werden: "Il faut commencer par accepter la responsabili-

8) Allocution du Général-Major Habyarimana Juvénal, Président de la République Rwandaise, sur la Dette Africaine, Addis Abeba, 30 novembre 1987. 
té de nos propres erreurs!"'. Im Falle von Madagaskar bedeutet dies, jene verfehlte Politik einer überstürzten Industrialisienung, wie sie Ende der siebziger Jahre propagiert wurde, nicht einfach im Zeichen neuer Gegebenheiten beiseite zu schieben und vergessen zu lassen, sondern sie offentlich und selbstkritisch zu hinterfragen: Welche naiven, allein an Äusserem orientierten Bilder von Entwicklung haben die damalige Politik geprăgt, und was ist aus dem Scheitern der früheren Plăne für die heutige Situation zu lernen? Wie weit haben die Madagassen selber im Glauben an billigen Fortschritt jene innere Würde verkauft, deren Verlust sie heute so bitter empfinden?

Aư den zweiten nötigen Schritt weist Habyarimana dort hin, wo er von den "responsabilités" spricht, "qui sont celles du monde de dehors." Ge Gerade auch im Blick auf unser Beispiel Madagaskar sind damit deutlich die internationalen Organisationen und die westlichen Industrielănder, inklusive die Schweiz, angesprochen. Denn es geht heute nicht nur darum, über jenen madagassischen Minister kopfschüttelnd nachzudenken, der 1979 innerhalb einer Woche 23 internationale Verträge über schlüsselfertige Industrien unterschrieben haben will. Es muss gleichermassen darum gehen, nach der Mitverantwortung jener zu fragen, die eine derartige Politik nicht nur mitgetragen, sondern im kurzfristigen Eigeninteresse auch massiv gefördert haben. Aus dieser Mitbetroffenheit kann sich die Schweiz so wenig wie andere Industriestaaten heraushalten.

Eines der dringlichsten entwicklungspolitischen Gebote zur Zeit der Schuldenkrise müsste also darin bestehen, die gemeinsame Verantwortung für eine gemeinsam geprägte, aber verfehlte frühere Politik gemeinsam anzuerkennen und die Folgerungen daraus gemeinsam durchzuarbeiten. Damit kăme - mehr als durch humanitäre Nothilfe oder soziale Ergănzungsprogramme zu den Strukturanpassungen - eine neue Beziehung zum Ausdruck, die das technizistische Paradigma durch eine entscheidende zusătzliche Dimension bereichern könnte. Rein quantitativ und materiell gesehen blieben dabei die Probleme gewiss dieselben. Aber etwas von der Würde, die Prăsident Habyarimana ansprach, wăre zurückgewonnen, damit aber auch eine der wichtigsten Voraussetzungen dafür, dass Madagaskar lăngerfristig zu einer eigenen Identităt und zu eigenen inneren Entwicklungskrăften findet.

Leider sieht es zur Zeit nicht so aus, als ob diese Art von innerer Verbindung und gemeinsamer Verantwortlichkeit bald zu einem wesentlichen Merkmal globaler Entwicklungspolitik werden könnte. Zu stark prăgt das alte Paradigma unser Denken und hindert uns, aus dem Bezug zu hintergründigen Wirklichkeiten neue Ideen für die Zusammenarbeit zu entwickeln. Aus diesem Zusammenhang verstehe ich den Satz eines madagassischen Ministers, der einmal im kleinen Kreis bemerkte:

"Parfois c'est mieux de ne pas coopérer, parce que cela laisse un goût amer."

9) a.a.O. S. 9 .

10) a.a.O. S. 8 . 


\section{Elne neue Suchrichtung: konvex und konkav}

Abschliessend nun zurück zur Frage, ob für die Weiterentwicklung der Entwicklungszusammenarbeit nicht ein neues Paradigma nơtig wăre - eine neue Suchrichtung nach dem, was sich in Entwicklung und Unterentwicklung abspielt.

In den vorangehenden Abschnitten habe ich diese Frage bejaht. Ich habe zu zeigen versucht, wie sich ein solches neues Paradigma von unseren gegenwărtigen Vorstellungen unterscheiden müsste: Es geht darum, ergănzend zu vielen ăusseren Systemelementen und Faktoren auch innere Wirkkraftte von Mensch und Gesellschaft wahrzunehmen. Anhand der Entwicklungsprobleme Madagaskars haben wir festgestellt, dass der seelische Hintergrund im Lebensganzen ebenso wirkmăchtig ist wie der von der Entwicklungszusammenarbeit gestaltete konkrete Vordergrund. In unserem Bemühen um ein angemessenes Verstăndnis der Realität müssen die beiden Ebenen zwar immer unterschieden werden; sie lassen sich aber weder trennen noch kausal-logisch miteinander vernetzen. Wir haben es mit unterschiedlichen, aber in unauflösbarer Verbundenheit wirkenden Aspekten einer einzigen Realität zu tun.

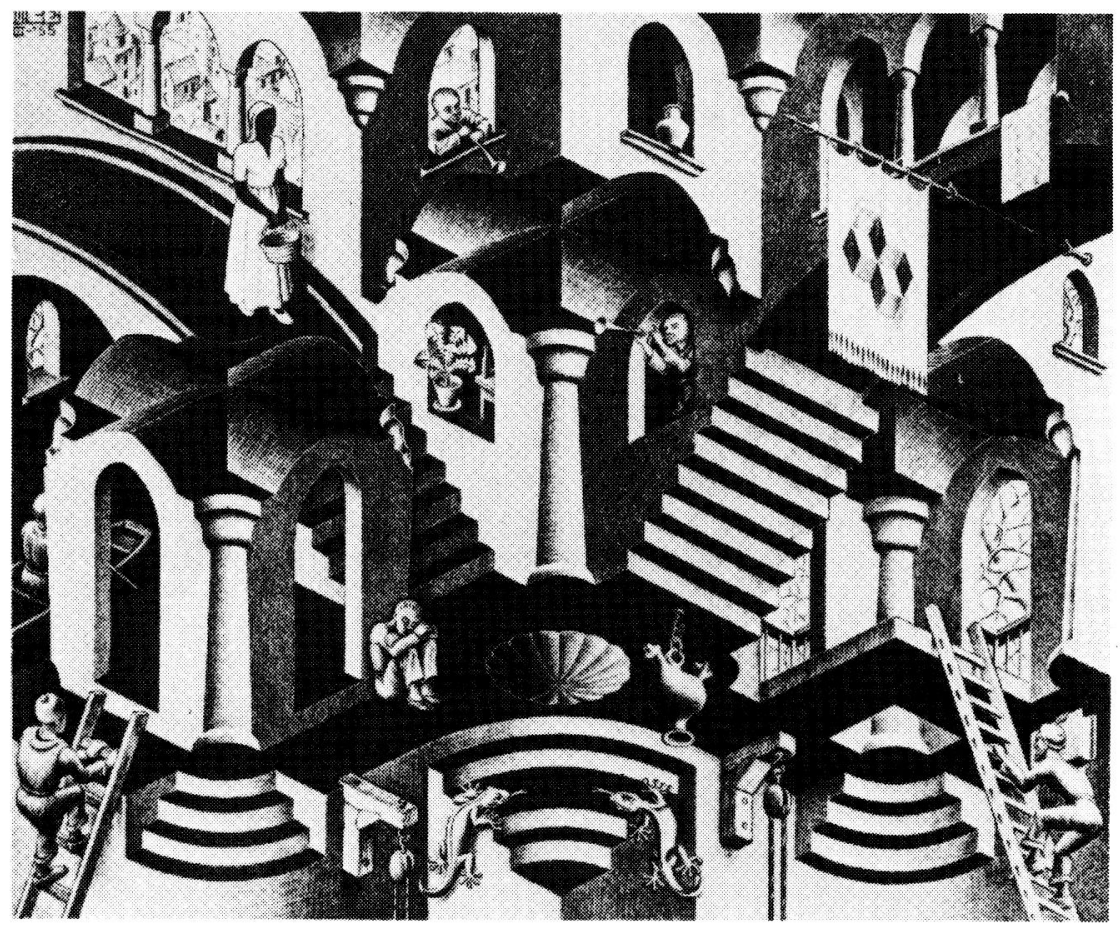


Für diese Komplementarităt habe ich beim holländischen Künstler M.C. Escher eine eindrückliche Illustration gefunden. In seiner berühmten Lithographie "Konvex und Konkav" aus dem Jahre 1955 schildert Escher einen merkwürdig verschachtelten Gebăudekomplex, dessen Raumsinn und Perspektive sprungartig wechselt, je nachdem der Betrachter seine eigene Optik auf das Ganze richtet: bald erscheinen ein und dieselben Gebăudeformen als konvex, bald als konkav. Es gibt keinen objektiven Maßstab, der sie eindeutig und ausschliesslich das konvex oder konkav erscheinen liesse. Die Wirklichkeit enweist sich als doppelgesichtig.

Konvex heisst "erhaben", "nach aussen gewölbt"; es kann für jene âussere Wirklichkeit stehen, die wir nach dem heutigen Paradigma durch Zergliederung und Vernetzung relativ leicht (und sehr effektiv!) in den Griff nehmen. Dazu gehören die naturrăumlichen Gegebenheiten, sozio-ökonomische Zusammenhänge, ethnisch-kulturelle Merkmale oder technische Gesetzmăssigkeiten.

Konkav bedeutet "vertieft", "nach innen gewölbt"; es ist für mich Ausdruck der inneren oder seelischen Wirklichkeit, die sich - wie wir gesehen haben - in unserem gegenwărtigen Paradigma so schlecht integrieren lăsst. Zu ihr gehören die unplanbaren seelischen Bedürfnisse des Menschen, seine Beziehungen zu Mitmenschen und das Geheimnis seiner künstlerischen, gedanklichen oder technischen Kreativität.

Eine neue Orientierung in der Entwicklungszusammenarbeit ist wohl nur zu gewinnen, wenn wir bereit sind, diesem anderen, nicht technizistisch integrierbaren Aspekt der Wirklichkeit jene Aufmerksamkeit zu schenken, die er angesichts seiner Wirksamkeit verdient. Die Entwicklung hat - wenn sie nachhaltig sein soll - eine innere Dimension, die unser bisheriges Paradigma sprengt. Die neue Suchrichtung spielt jedoch nicht nur auf diese zusätzliche Dimension, sondern darauf, die konkave mit der konvexen Realităt zu verbinden, so wie Escher das in seiner enwähnten Lithographie anstrebt. Das neue Paradigma besteht nicht einfach in einer neuen Perspektive. Es fordert vielmehr unsere Bereitschaft zu ständigem Perspektivenwechsel.

Ich gebe zu, dass dies keine bequeme Aussicht ist, und ich bin mir bewusst, dass der Umgang mit dem neuen Paradigma in der Praxis noch viele Fragen aufwirt, die hier nicht angesprochen worden sind. Deshalb habe ich schon zu Beginn betont, dass mit dem neuen Paradigma eher eine neue Suchrichtung als ein fertiges Konzept gemeint ist - eine Suchrichtung allerdings, ohne die ich mir mein eigenes Engagement in der Entwicklungszusammenarbeit nicht mehr vorstellen kann. 